\section{Pioneering Jupiter meeting at RAS}

\section{by John Gribbin}

ON Friday May 24 the Royal Astronomical Society broke new ground with their first meeting devoted entirely to discussion of the planet Jupiter. The stimulus to hold such a meeting at present came, of course, from the historic data gathered by Pioneer 10 a few months ago, and which are now being analysed. The process of analysis and interpretation is a long one, and although some of the speakers at the meeting are, it seems, regular acquaintances from similar meetings held at various institutions every few weeks, they have yet to hear each other covering entirely familiar ground. So what emerged from the recent RAS meeting was not in any sense a definitive statement about the nature of Jupiterrather, a progress report of the state of the art, which raises one or two extremely puzzling questions.

The meeting began with a resume from K. Runcorn (University of Newcastle upon Tyne) of the pre-Pioneer picture of Jupiter and its radioactivity. It is now some 52 years since Jeffreys produced the first model of Jupiter, Runcorn recalled, and 37 years since advances in quantum mechanics showed that metallic hydrogen can be formed at pressures comparable to those found in the interior of that giant planet. For some two decades the presence of radio emission from Jupiter has been known -indeed it is the brightest object in the radio sky - and this ties in well with the idea of a fluid metallic core in which dynamo processes operate.

No discussion of Jupiter is complete without mention of the Great Red Spot, which rotates with a period close to $9 \mathrm{~h} 55 \mathrm{~min} 40 \mathrm{~s}$, some $10 \mathrm{~s}$ more than the rotation period of radio features. This can be explained, broadly speaking, by Hide's model which Runcorn called "the most physically satisfying explanation of the Great Red Spot". In this model, the spot is seen as the visible part of a Taylor column produced by an irregularity (mountain or crater) in the underlying solid surface. Then, the spot period represents the rotation period of the solid mantle, and the radio period the period of the weakly coupled core.

If that is the case, it is to be expected that slight variations in the two periods - a few milliseconds or so-could be correlated in much the same way that variations in the rotation of the Earth's magnetosphere can be correlated with changes in the length of day. Tantalisingly, the available radio observations can neither confirm nor deny this hypothesis, although to my eye the curves presented by Runcorn seem to fit this proposal better than the alternative hypothesis, that the radio period is strictly constant. One thing the Earthbound radio observations do imply is that the Jovian magnetic field is, if dipolar, inclined at about $11^{\circ}$ to the rotation axis-and this provides a neat tie in with the Pioneer 10 data.

E. J. Smith (Jet Propulsion Laboratory, CalTech) described the Pioneer observations of the Jovian magnetic field. The data are not sufficient to define a unique model, but the simplest model which fits them is of a dipolar field, inclined at $11^{\circ}$ and offset from the planet's centre, with a strength of 4 gauss at the equator. Closest approach of the vehicle to Jupiter was 2.8 Jovian radii, and the field showed a steady cube law dependance on distance for most of the trajectory, as expected for a dipolar field. The offset of the dipole from the centre of Jupiter seems to be about one tenth of the planet's radius $\left(R_{\mathrm{J}}\right)$, much more than the equivalent offset of the Earth's field.

The magnetosphere beyond some 20 $R_{\mathrm{J}}$ is strongly deformed from the dipolar form which is characteristically closer to the planet, and seems to contain a thin current sheet. This has an important effect on the distribution of energetic particles around Jupiter, as J. Simpson (Enrico Fermi Laboratory, University of Chicago) and R. W. Fillius (University of California, San Diego) reported.

Much of the data concerning the particle distributions and the sweeping up effect of some of the Jovian satellites is already familiar from published work, and the latest developments add little to the earlier analyses. But two intriguing features remain to be explained. First, the distribution of both protons and non-relativistic electrons peaks slightly further out from Jupiter than the Pioneer 10 periapsis. Extrapolation of the measurements suggests that there is a 'particle hole' close to the planet, and this can hardly be attributed to the influence of Amalthea since high energy ( $35 \mathrm{MeV}$ ) electrons are not affected. Pioneer 11 is now targeted to fly through this hole, so its reality should soon be confirmed. If the distribution of protons does, however, peak again close in to Jupiter then Pioneer 11 is unlikely to survive.

But the most intriguing discovery mentioned at the RAS meeting concerns the bursts of relativistic electrons apparently ejected by Jupiter. These bursts were first detected by Pioneer 10 half a year before encounter, and got bigger as the spacecraft approached the planet. Clearly they are not solar particles, since they come from the wrong direction; the idea that they might represent interstellar particles leaking in to the Solar System from outside held some appeal at one time, but now it seems clear that they do originate from Jupiter.

The crucial evidence is that once Pioneer 10 had made its dive through the Jovian magnetosphere it was possible to extrapolate back from observations of the roughly $10 \mathrm{~h}$ periodic variations produced by the rotation of the planet. This extrapolation showed that all the bursts seen on the way to Jupiter showed structure on a timescale of $10 \mathrm{~h}$ exactly in phase with the variations inside the Jovian magnetosphere. Clearly, unless Pioneer 10 had travelled in a trajectory specially favoured for particle ejection (which seems most unlikely), Jupiter must be radiating waves of particles outwards over a wide range of latitudes. This is very disturbing for established theories which suggest that particles should be nicely contained in the equatorial plane, and shows how much remains to be discovered about Jupiter. At the same time, this source of non-solar particles provides an invaluable new tool for investigating propagation of particles in interplanetary space. With Pioneer 11 still to make its (perhaps suicidal) close dive past Jupiter, it looks as if this will not be the last RAS meeting devoted exclusively to the giant of the Solar Svstem.

\section{East African grasses}

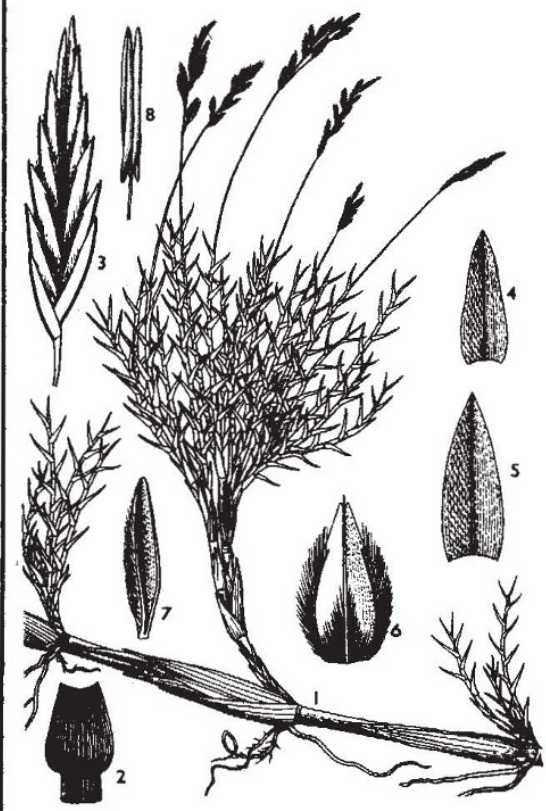

The second part of the Graminae section of the Flora of Tropical East Africa (edit. by Clayton, Phillips and Renvoize; Crown Agents of Oversea Governments and Administrations, London, April 1974, £2.75) contains this drawing of Odyssea paucinervis which is found south of the Congo River on saline soils between 1,800 and $1,900 \mathrm{~m}$ 